\title{
Valor del Uretrocistograma con cadena en el tratamiento de la Incontinencia Urinaria de Esfuerzo.
}

\section{MATERIAL Y METODOS}

Doctores:

* Alberto Angulo Roa

** Germán Uriza Gutiérrez

*** Rafael Perdomo Perdomo

**** Germán Sabogal Nariño

$* * * * *$ Hugo Márquez

\section{INTRODUCCION}

La frecuencia de la incontinencia de orina en la mujer, hace de esta afección un tema de permanente actualidad, ya que del conocimiento de la fisiopatología y de los medios de diagnóstico, depende en gran parte la adecuada conducta terapéutica.

Muchas han sido las definiciones propuestas para este síndrome génito urinario. Nosotros consideramos como la más razonable la siguiente: La Incontinencia Urinaria de Esfuerzo (IUE) es el escape involuntario de orina cuando la presión intravesical es mayor que la presión uretral máxima, situación que se presenta con la relajación de las estructuras de sostén de la uretra y de la vejiga, sin que exista actividad del detrusor. Este escape de orina puede ser ocasionado al toser, estornudar, pujar, correr, ante los cambios de postura, etc.

Con base en extensos estudios se ha concluido que la presencia de un ángulo uretrovesical posterior normal y un eje uretral conservados, son esenciales para el mecanismo de la continencia urinaria, y que en pacientes con IUE están alterados en forma característica tanto el uno como el otro.

Jefe de 1 unidad de Ginecologia

Profesor Asistente Departamento de Ginecología y Obstetricia

Residente II Departamento de Ginecología y Obstetricia

Residente II Departamento de Ginecología y Obstetricia

Residente I Departamento de Radiología.

Universidad Javeriana
A todas las pacientes que acudieron a la consulta externa del Hospital San Ignacio y del Centro de Salud No. 41 de Bogotá, a partir de enero de 1978 y a consultar por incontinencia urinaria, se les formuló un interrogatorio para confirmar el diagnóstico clínico de IUE. El interrogatorio estuvo formado por las siguientes cuatro preguntas:

1. En cuál de estas posiciones aparece pérdida de orina con los esfuerzos?

a) Decúbito supino?

b) Decúbito lateral?

c) Bipedestación?

La respuesta debe ser: EN TODAS LAS POSICIONES.

2. La expulsión de la orina es en pequeñas cantidades?

La respuesta debe ser: SI.

3. La expulsión de la orina es inmediata con el esfuerzo, o hay un intervalo libre que oscila entre 10 y 15 segundos entre el esfuerzo y la expulsión de la orina?

La respuesta debe ser: INMEDIATA CON EL ESFUERZO.

4. Una vez iniciada la emisión de orina, es capaz de frenarla voluntariamente?

La respuesta debe ser: SI.

Hecho el diagnóstico clínico, se remitieron las pacientes a la consulta especializada de IUE que funciona en nuestro Hospital desde hace diez años. Con el fin de descartar patología asociada a la IUE, se les practicaron a todas las pacientes: Parcial de orina, urocultivo, calibración uretral, cistoscopia y Prueba de Marshall. 
Descartada la patología asociada, quedaron para estudio treinta $\mathrm{y}$ un pacientes, todas con prueba de Marshall positiva y a las cuales se les solicitó Uretrocistograma con cadena preoperatorio, con el fin de clasificar el tipo de incontinencia urinaria de esfuerzo, y Uretrocistograma post-operatorio para verificar la corrección de los ángulos uretro vesicales.

Técnica del Uretrocistograma con cadena:

Es un procedimiento radiográfico que permite visualizar la uretra y la vejiga, mediar los ángulos uretrovesicales anterior y posterior y el descenso vesical. Este procedimiento data del año de 1953.

Nosotros utilizamos la siguiente técnica:

Para visualizar la vejiga se usa como medio de contraste una mezcla de 150 cc. compuesta por $110 \mathrm{cc}$. de solución salina y 40 cc. de solución acuosa yodada. (Uromiron Shering AG).

Para la visualización uretral se utiliza una cadena de cuentas metálicas cuya longitud es de 20 centímetros, la cual se inserta en la uretra con la ayuda de un hemisonda de Nelatón No. 16, que se remueve fácilmente una vez se haya confirmado que el extremo de la cadena se encuentra en la vejiga; la mesa de Rayos se coloca en posición vertical, los pies de la paciente separados 30 centímetros para prevenir obstáculos en el descenso de la uretra y la vejiga. Se instruye a la paciente para practicar un incremento de la presión intraabdominal y se toman una proyección anteroposterior, y otra lateral en el momento de máximo esfuerzo.

Interpretación del Uretrocistograma:

Enlas proyecciones laterales y en el momento de máximo esfuerzo, se efectúan las siguientes medidas:

a) Angulo Uretro Vesical posterior (AUVP), el cual se encuentra formado por el eje de la uretra y el segmento proximal de la base vesical, cuyo valor normal no debe exceder de 100 grados. b) Angulo Uretral Anterior (AUVA) formado por el eje de la uretra y una perpendicular al piso. Su valor máximo es de 30 grados.

En la proyección antero posterior se valora el descenso vesical, midiendo la distancia entre la base de la vejiga y el borde superior de la sínfisis púbica, la cual no debe exceder de 2,5 centímetros.

De acuerdo con el Uretrocistograma con cadena, las anormalidades uretroversicales se clasifican en tres grupos:

TIPO A, en el cual el ángulo uretrovesical posterior es mayor de 100 grados y el uretral anterior es normal.

TIPO B, el ángulo uretrovesical posterior es mayor de 100 grados y el uretral anterior es mayor de 30 grados.

TIPO C, en el cual el ángulo uretrovesical posterior es normal, pero el ángulo uretral anterior es mayor de 30 grảdos. (Gráfica 1).

En los Tipos A y B se presenta incontinencia urinaria de esfuerzo. En el tipo C hay continencia, pero se considera a este grupo de pacientes potencialmente incontinentes, siendo éste un hallazgo posterior a la cirugía correctora de la IUE, bien sea por fracaso quirúrgico parcial en una técnica adecuada, o porque se empleó un método quirúrgico inadecuado para el tipo de incontinencia.

\section{Técnica quirúrgica:}

Sin duda la clave de una intervención satisfactoria para la corrección de la IUE radica en la elevación retropública de la unión uretrovesical, por encima del nivel más inferior de la cara posterior de la base de la vejiga, sea cual fuere la vía de acceso utilizada: vaginal o abdominal.

A las pacientes con Uretrocistograma TIPO A se les intervino con operación de Kelly, exceptuando el caso número 15; a esta paciente se le practicó histerectomía abdominal y Marshall por presentar patología gine- 


$$
\bar{D}
$$




$$
\frac{D}{D}
$$


cológica asociada a la IUE. Creemos que cuando se utiliza la vía abdominal por una patología ginecológica asociada a la IUE, ésta debe corregirse con la operación de Marshall sin importar el tipo de IUE.

A las pacientes con Uretrocistograma TIPO B se les practicó operación de Marshall, exceptuando el caso número 31 . A esta paciente se le realizó histerectomía vaginal y operación de Kelly, debido a la necesidad de acortar el tiempo quirúrgico por el mal estado de la paciente.

\section{RESULTADOS}

Edad:

Es importante comentar que antes de los 30 años no se presentó caso alguno de IUE. La mayor incidencia se encontró entre los 41 y los 50 años y correspondió al $38.7 \%$. (Gráfica 2).
Paridad :

Como era de esperarse, se encontró la mayor incidencia de IUE en las multíparas $(41.9 \%$ ) y en las grandes multíparas (38.7 \%). No se encontró diferencia significativa entre estos dos grupos de pacientes, pero sí una marcada diferencia entre estos grupos y los de las primigestantes $(12.9 \%$ y las nulíparas $(6.4 \%)$. (Gráfica 3 ).

\section{Celes:}

Se encontró IUE en pacientes con diferentes grados de cistocele y en dos casos sin cistocele, apoyando nuestra afirmación de que el descenso vesical no es de importancia directa en la génesis de la IUE. Se encontró IUE en cualquier grado de Uretrocele, pero no encontramos afección sin Uretrocele.

Cuando las celes de uretra y vejiga son gra-

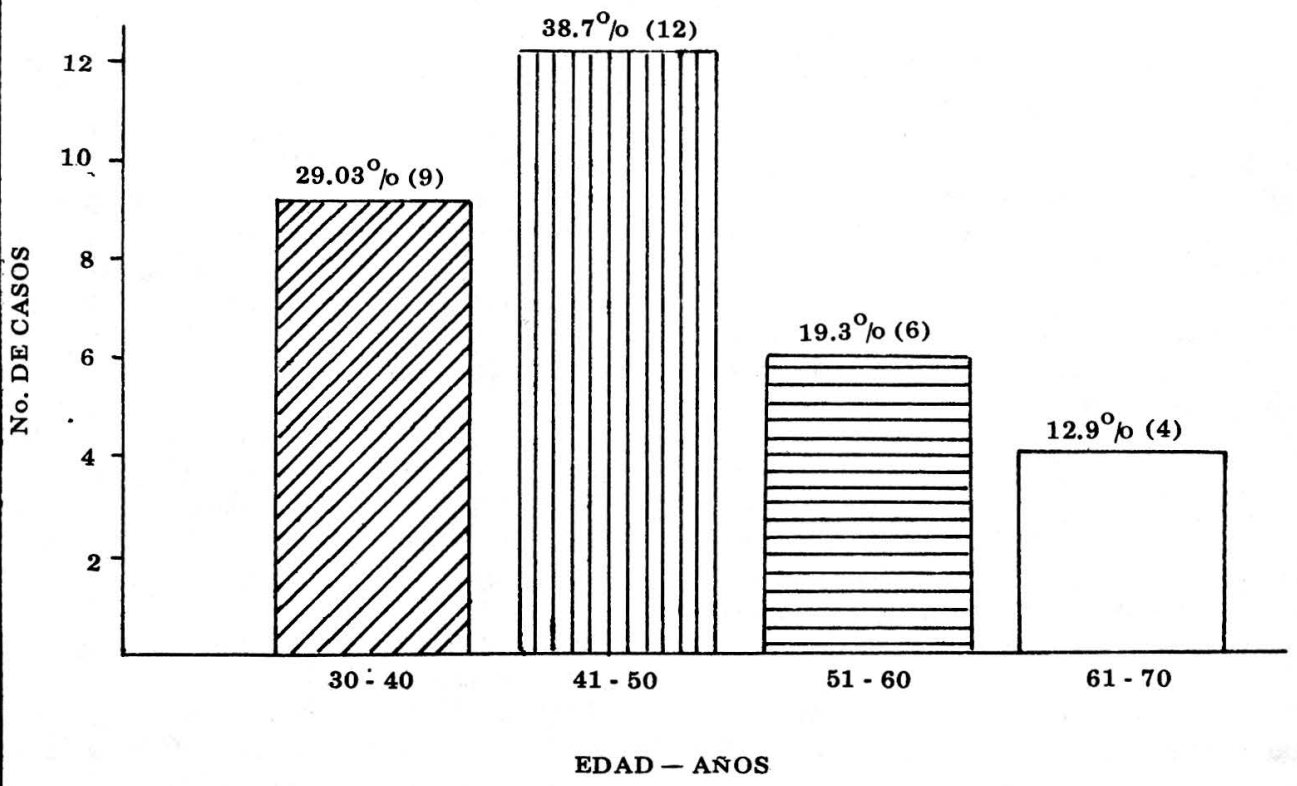

GRAFICA No. 2 


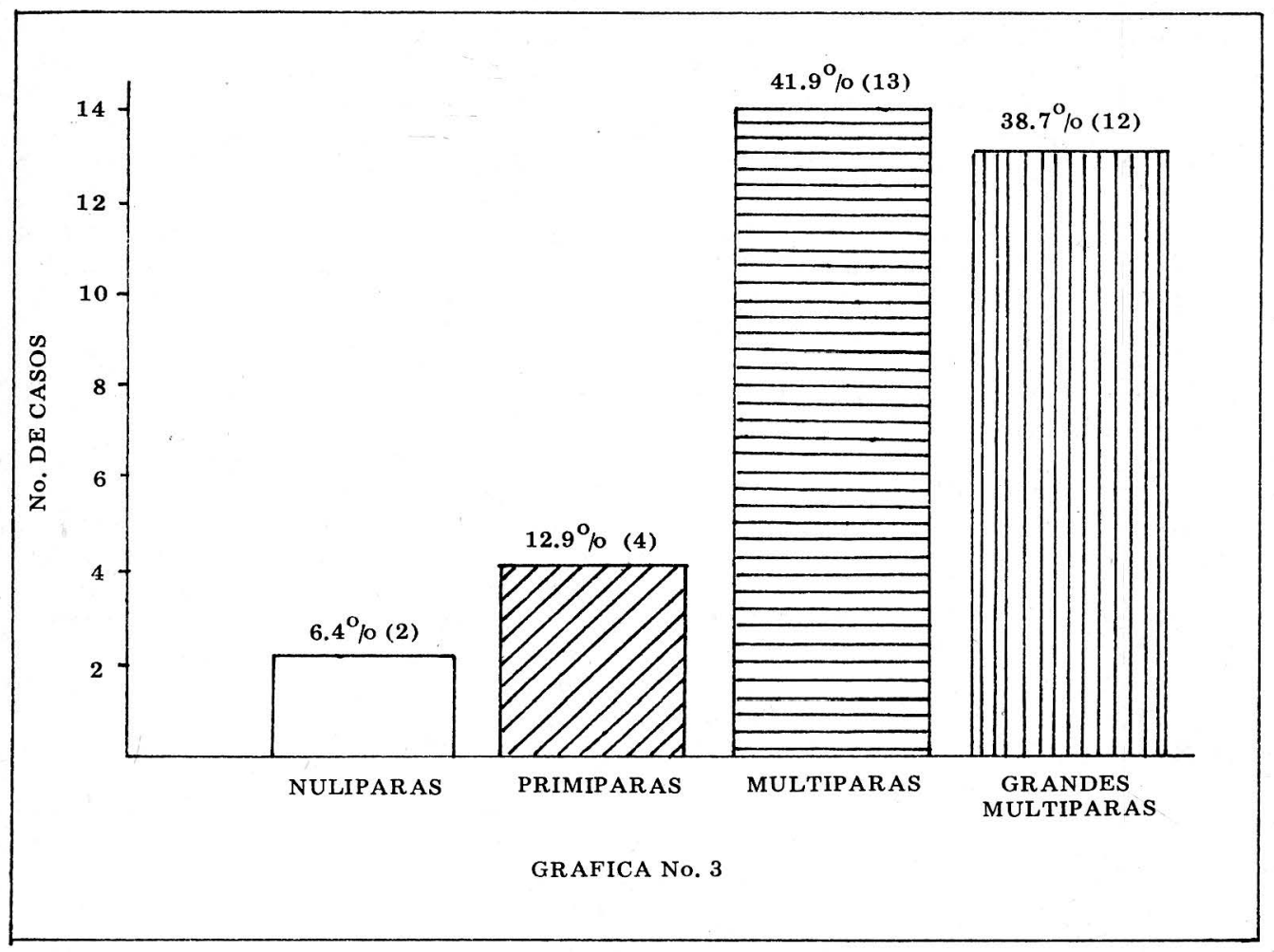

do III, la IUE no es el síntoma predominante, sino que, por el contrario, es raro encontrar dicho sintoma en estos grados extremos.

La presencia de rectocele, desgarro perineal o histerocele no inciden en la génesis de la IUE. (Cuadro No. 1).

\section{Uretrocistograma:}

Para valorar el éxito o el fracaso de la inter. vención, se les tomó a todas las pacientes uretrocistograma pre y post-operatorio, con los siguientes hallazgos:

El uretrocistograma preoperatorio muestra que la mayoría de la IUE son de TIPO B, con 26 casos que constituyen el $83.8 \%$. Las pacientes con uretrocistograma postoperatorio TIPO A. ( 2 casos) y TIPO B. (4 casos) continúan con IUE $(19.3 \%)$. Las 25 pacientes con uretrocistograma post-opera- torio normal y TIPO C. $(80.6 \%)$ están actualmente continentes y su seguimiento ha sido de un año a partir de la fecha de la intervención. Consideramos potencialmente incontinentes 4 casos, por presentar uretrocistograma post-operatorio TIPO C (12.9 $\%$ ). (Cuadro 2).

En las 6 pacientes con persistencia de IUE, la técnica quirúrgica no corrigió los ángulos hasta el límite de la normalidad, sin que se haya modificado el tipo de IUE. (Casos 7 12-17-18-28-31).

En las 4 pacientes con uretrocistograma post-operatorio TIPO $\mathrm{C}$, la cirugía cambió el tipo de uretrocistograma del TIPO A. al TIPO C. en un caso, y del TIPO B. al TIPO C. en 3 casos. (Casos 3-4-11-25).

Los fracasos de la operación de Marshall fueron 3 . en 26 intervenciones y corresponden a un 13\% (Marchall-Marchetti y Kran- 
GRADOS DE CELES

\begin{tabular}{|l|c|c|c|c|c|c|c|c|}
\hline GRADOS & \multicolumn{2}{|c|}{ SIN } & \multicolumn{2}{c|}{ I } & \multicolumn{2}{c|}{ II } & \multicolumn{2}{c|}{ III } \\
\hline & $\begin{array}{c}\text { No. } \\
\text { CASOS }\end{array}$ & $\%$ & $\begin{array}{c}\text { No. } \\
\text { CASOS }\end{array}$ & $\%$ & $\begin{array}{c}\text { No: } \\
\text { CASOS }\end{array}$ & $\%$ & $\begin{array}{c}\text { No. } \\
\text { CASOS }\end{array}$ & $\%$ \\
\hline URETROCELE & 0 & 0 & 8 & 25.8 & 21 & 67.7 & 2 & 6.4 \\
\hline CISTOCELE & 2 & 6.4 & 7 & 22.5 & 21 & 67.7 & 1 & 3.2 \\
\hline RECTOCELE & 3 & 9.6 & 6 & 19.3 & 21 & 67.7 & 1 & 3.2 \\
\hline D.P. & 5 & 16.1 & 3 & 9.6 & 23 & 74.1 & 0 & 0 \\
\hline HISTEROCELE & 0 & 0 & 0 & 0 & 1 & 3.2 & 0 & 0 \\
\hline
\end{tabular}

CUADRO No. 1

URETROCISTOGRAMA

\begin{tabular}{|l|c|c|c|c|}
\hline \multirow{2}{*}{ TIPO } & \multicolumn{2}{|c|}{ PRE-OPERATORIO } & \multicolumn{2}{c|}{ POST-OPERATORIO } \\
\hline & No. & $\mathrm{o}_{\mathrm{o}}$ & No. & $\mathrm{o}_{\mathrm{O}}$ \\
\hline NORMAL & 0 & 0 & 21 & 67.7 \\
\hline TIPO A & 5 & 16.12 & 2 & 6.4 \\
\hline TIPO B & 26 & 83.8 & 4 & 12.9 \\
\hline TIPO C & 0 & 0 & 4 & 12.9 \\
\hline
\end{tabular}

CUADRO No. 2

tz, 11\%). (Casos números 7-8-28).

Los fracasos de la operación de Kelly fueron 2 en 4 intervenciones, es decir, un 50 $\%$ (Hodgkinson $45 \%$ ). (Casos 12-17). En un caso de fracaso no se utilizó la técnica adecuada para el tipo de incontinencia (Caso $31-$ ), por lo cual no puede considerarse como fracaso de la técnica.

En la paciente con uretrocistograma preoperatorio de TIPO A, que pasó a TIPO C en el postoperatorio, intervenida con operación de Kelly, consideramos que el ángulo posterior fue extremadamente corregido, lo cual alteró el eje uretral y de este modo modificó el ángulo anterior que era normal (Caso 3-).

En las pacientes con uretrocistograma preoperatorio TIPO B, que pasaron a TIPO $\mathrm{C}$ en el post-operatorio, y a quienes se les practicó operación de Marshall, ésta corrigió aceptablemente el ángulo posterior y' en forma inadecuada el anterior. (Casos 4-1125). (Cuadro 3).

\section{CONCLUSIONES}

El presente trabajo permite concluir que: PRIMERO:

El uretrocistograma con cadena tiene valor, 
CUADRO No. 3

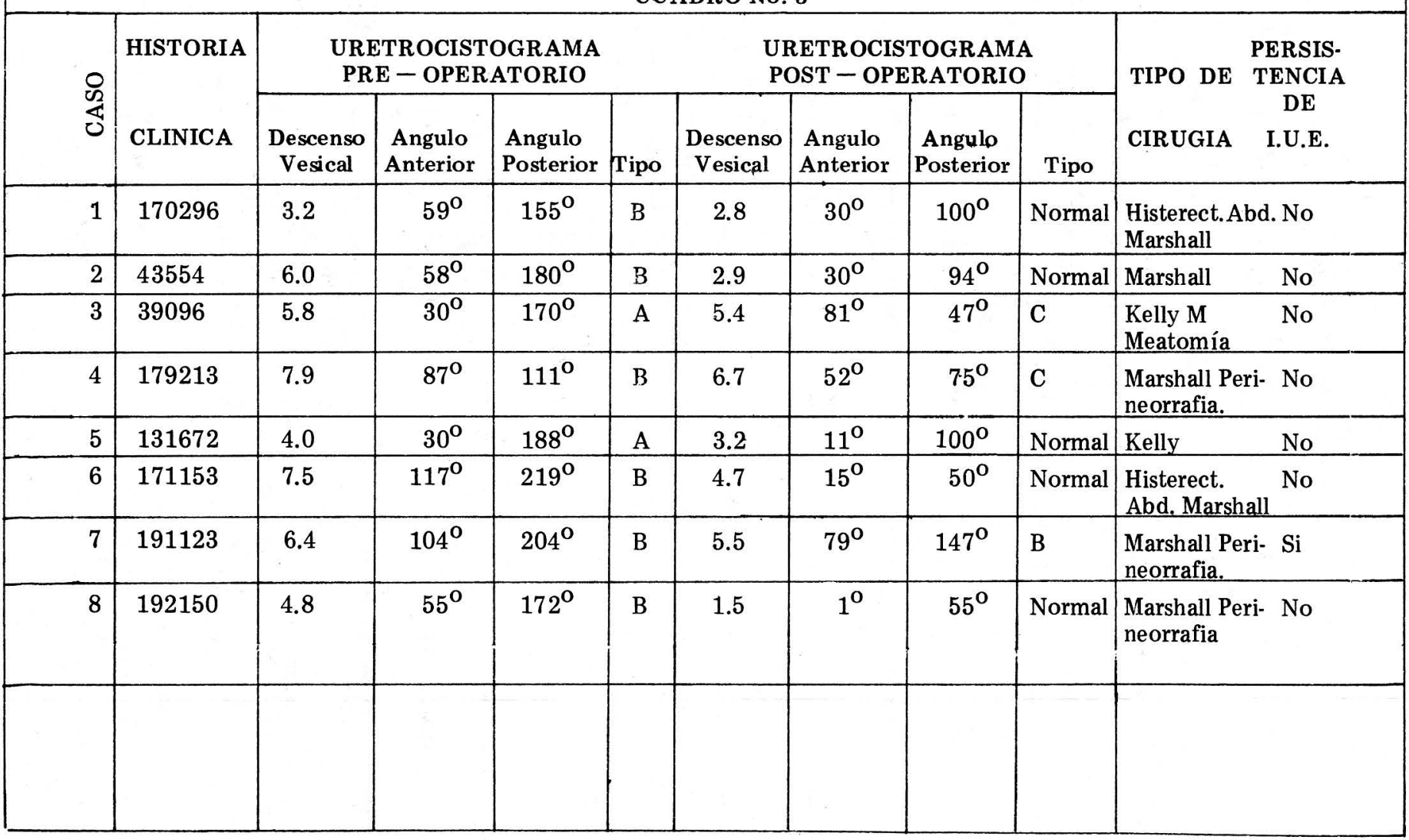




\begin{tabular}{|c|c|c|c|c|c|c|c|c|c|c|c|}
\hline & \multirow{3}{*}{$\begin{array}{l}\text { HISTORIA } \\
\text { CLINICA }\end{array}$} & \multicolumn{8}{|c|}{ CUADRO No. 3 (Cont.) } & \multirow{3}{*}{$\begin{array}{l}\text { TIPO DE } \\
\text { CIRUGIA }\end{array}$} & \multirow{3}{*}{$\begin{array}{c}\text { PERSIS } \\
\text { TENCIA } \\
\text { DE } \\
\text { I.U.E. }\end{array}$} \\
\hline & & \multicolumn{4}{|c|}{$\begin{array}{l}\text { URETROCISTOGRAMA } \\
\text { PRE - OPERATORIO }\end{array}$} & \multicolumn{4}{|c|}{$\begin{array}{l}\text { URETROCISTOGRAMA } \\
\text { POST - OPERATORIO }\end{array}$} & & \\
\hline & & \begin{tabular}{|l|} 
Descenso \\
Vesical \\
\end{tabular} & $\begin{array}{c}\begin{array}{c}\text { Angulo } \\
\text { Anterior }\end{array} \\
\end{array}$ & \begin{tabular}{|l|} 
Angulo \\
Posterior \\
\end{tabular} & Tipo & \begin{tabular}{|c|} 
Descenso \\
Vesical \\
\end{tabular} & $\begin{array}{r}\text { Angulo } \\
\text { Anterior } \\
\end{array}$ & $\begin{array}{c}\text { Angulo } \\
\text { Posterior }\end{array}$ & Tipo & & \\
\hline 9 & 194557 & 8.3 & $110^{\circ}$ & $194^{\circ}$ & B & 5.9 & $3^{\circ}$ & $60^{\circ}$ & Normal & $\begin{array}{l}\text { Marshall - Peri- } \\
\text { neorrafia. }\end{array}$ & No \\
\hline 10 & 157197 & 7.1 & $85^{\circ}$ & $150^{\circ}$ & B & 2.8 & $22^{\circ}$ & $82^{\circ}$ & Normal & $\begin{array}{l}\text { Histerectomía } \\
\text { Abd. Marshall }\end{array}$ & No \\
\hline 11 & 62861 & 7.0 & $57^{\circ}$ & $152^{\circ}$ & B & 7.0 & $40^{\circ}$ & $94^{\circ}$ & C & Marshall & No \\
\hline 12 & S. H. & 0.4 & $1^{0}$ & $120^{\circ}$ & $\mathbf{A}$ & 0.9 & $5^{\circ}$ & $138^{\circ}$ & A & Kelly & $\mathrm{Si}$ \\
\hline 13 & 195456 & 3.6 & $45^{\circ}$ & $176^{\circ}$ & B & 2.3 & $0^{\circ}$ & $67^{\circ}$ & Normal & $\begin{array}{l}\text { Marshall - Peri- } \\
\text { neorrafia. }\end{array}$ & No \\
\hline 14 & 198362 & 7.4 & $120^{\circ}$ & $171^{\circ}$ & $\mathrm{B}$ & 5.9 & $11^{\circ}$ & $74^{\circ}$ & Normal & Marshall & No \\
\hline 15 & 191964 & 1.1 & $3^{0}$ & $140^{\circ}$ & $\mathbf{A}$ & 1.6 & $30^{\circ}$ & $70^{\circ}$ & Normal & \begin{tabular}{|l|} 
Histerectomía \\
Abd.Marshall \\
\end{tabular} & No \\
\hline 16 & 170209 & 4.8 & $117^{\circ}$ & $207^{\circ}$ & $\mathrm{B}$ & 5.0 & $13^{\circ}$ & $44^{\circ}$ & Normal & Marshall & No \\
\hline 17 & 53667 & 6.8 & $5^{0}$ & $148^{\circ}$ & A & 4.1 & $30^{\circ}$ & $185^{\circ}$ & A & Kelly & $\mathrm{Si}$ \\
\hline 18 & S. H. & 7.0 & $78^{\circ}$ & $148^{\circ}$ & $\mathrm{B}$ & 7.2 & $64^{\circ}$ & $130^{\circ}$ & B & \begin{tabular}{|l|} 
Marshall \\
\end{tabular} & $\mathrm{Si}$ \\
\hline 19 & S. H. & 4.3 & $69^{\circ}$ & $156^{\circ}$ & B & 2.9 & $10^{\circ}$ & $58^{\circ}$ & Normal & Marshall & No \\
\hline 20 & 197885 & 3.0 & $52^{\circ}$ & $180^{\circ}$ & B & 1.7 & $10^{\circ}$ & $89^{\circ}$ & Normal & \begin{tabular}{|l|} 
Histerectomía \\
Abd. Marshall \\
\end{tabular} & No \\
\hline 21 & 196222 & 5.7 & $62^{\circ}$ & $174^{\circ}$ & B & 3.1 & $7^{\circ}$ & $59^{\circ}$ & Normal & \begin{tabular}{|l|}
$\begin{array}{l}\text { Marshall Peri- } \\
\text { neorrafia }\end{array}$ \\
\end{tabular} & No \\
\hline 22 & 193855 & 5.6 & $91^{\circ}$ & $182^{\circ}$ & B & 4.0 & $8^{0}$ & $45^{\circ}$ & Normal & Marshall Peri- & No \\
\hline 23 & 124161 & 1.5 & $46^{\circ}$ & $154^{\circ}$ & B & 1.8 & $12^{\circ}$ & $66^{\circ}$ & Normal & \begin{tabular}{|l|}
$\begin{array}{l}\text { Marshall Peri- } \\
\text { neorrafia }\end{array}$ \\
\end{tabular} & No \\
\hline 24 & S. H. & 1.7 & $66^{\circ}$ & $165^{\circ}$ & B & 1.5 & $20^{\circ}$ & $96^{\circ}$ & Normal & Marshall & No \\
\hline
\end{tabular}


CUADRO No. 3 (Cont.)

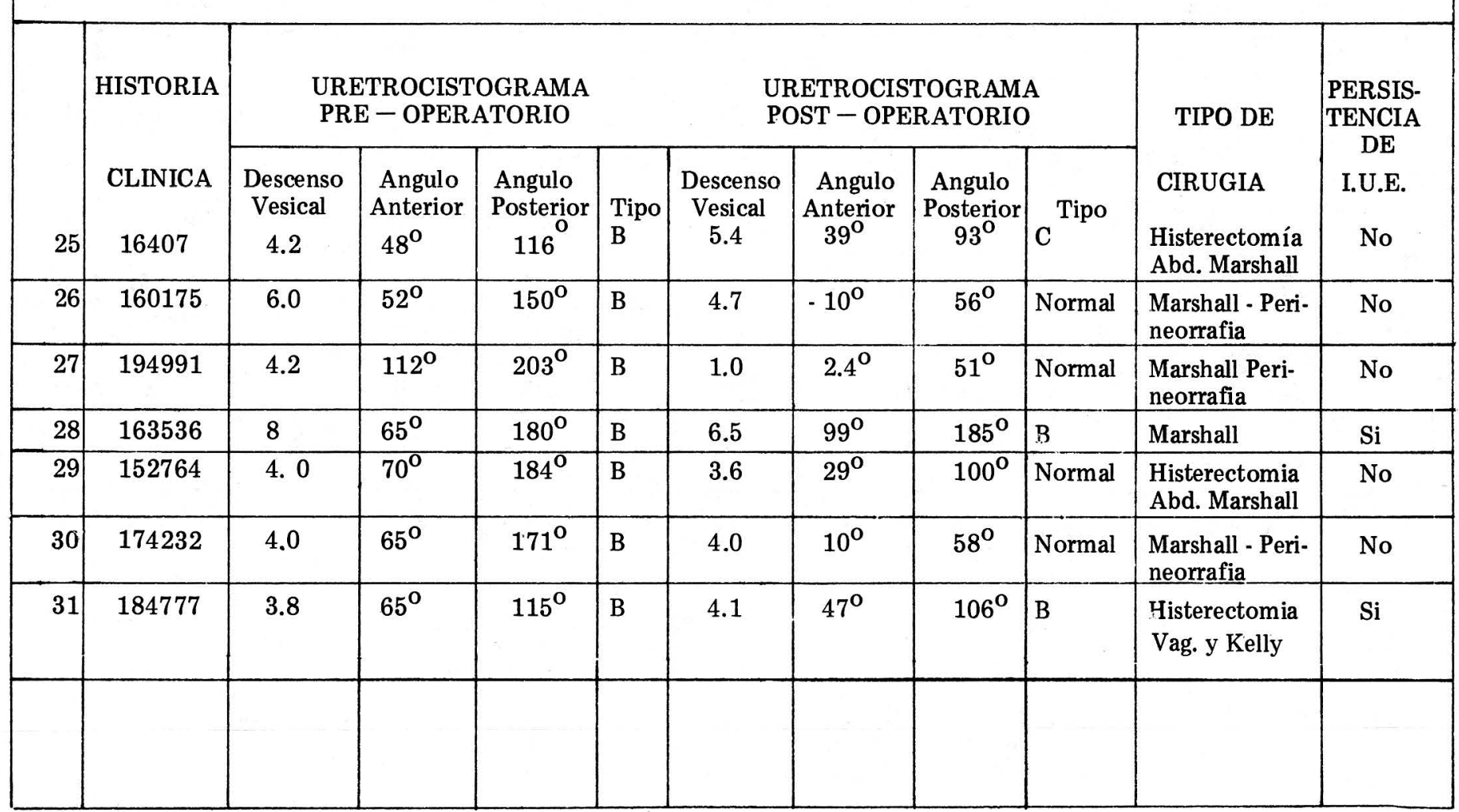


aunque no absoluto, en el dianóstico de la incontinencia urinaria de esfuerzo, hecho éste demostrado por las alteraciones de los ángulos en la totalidad de las pacientes estudiadas.

El uretrocistograma con cadena permite confirmar el diagnóstico de una incontinencia urinaria de esfuerzo, peró no descarta que exista concomitantemente otra entidad productora de incontinencia urinaria.

Para poder llegar a un diagnóstico exacto de incontinencia urinaria de esfuerzo es necesaria, además, la realización de una historia clínica completa, que incluya interrogatorio y examen físico. La disponibilidad de investigaciones urodinámicas, de ninguna manera debe sustituir al interrogatorio y a la exploración física, que siguen siendo un aspecto central en el estudio de estas pacientes.

\section{SEGUNDO:}

El uretrocistograma con cadeña nos muestra qué tipo de alteración anatómica uretrovesical existe, y en esta forma permite la clasificación de la incontinencia urinaria de esfuerzo como TIPO A. o TIPO B.

\section{TERCERO :}

Confirmamos la teoría de que la restauración de un ángulo uretrovesical posterior es el hecho más importante común a todos los casos de reconstrucción quiriurgica y en la incontinencia urinaria de esfuerzo. Fallas de recurrencia tienen una asociación invariable con la imposibilidad para crear o mantener un ángulo uretrovesical posterior adecuado, hecho confirmado en los uretrocistogramas post-operatorios de los seis casos de recurrencia presentados en nuestro medio.

\section{CUARTO:}

Hemos encontrado que la localización de cuello vesical y la uretra proximal con relación a la sínfisis, no son de importancia directa en la génesis de la IUE, ya que muchas pacientes con descenso marcado de la vejiga (cistocele) observado en el uretrocistograma post-operatorio, y en quienes los ángulos uretrovesical posterior: y ejè uretral se conservan, son continentes.

\section{QUINTO:}

El uretrocistograma con cadena permite elegir la técnica quirúrgica adecuada para la IUE Para el TIPO: A, operación de Kelly, y para el TIPO B, operación de Marshall.

\section{SEXTO:}

La continencia urinaria TIPO C, demostrada por el uretrocistograma post-operatorio, es una creación iatrogénica, ya que ninguna de nuestras pacientes tenía este tipo de uretrocistograma antes de la intervención. Cuatro casos de nuestro estudio se encuentran en esta clasificación y la totalidad son actualmente continentes.

\section{SEPTIMO :}

En los casos deIUE TIPO A asociada con patología ginecológica, que requiere intervención quirúrgica por vía abdominal, se les practicará operación de Marshall, puesto que esta técnica puede corregir tanto el ángulo uretrovesical solo, o éste y el eje uretral.

\section{oCTAVO:}

Estimamos que la operación más adecuada para la corrección de la IUE es ałgún tipo de uretropexia retropúbica, para nosotros la operación de Marshall.

\section{NOVENO:}

El uretrocistograma no es, en verdad, indispensable para el diagnóstico de IUE; pero la información adicional que brindan los estudios radiográficos pre y post-operatorios, justifican el esfuerzo y el costo, ya que propician la institución de un tratamiento-apropiado y el pronóstico de la corrección quirúrgica.

\section{RESUMEN}

La frecuencia de la incontinencis de orina 
en la mujer hace de esta afección un tema de permanente actualidad, ya que del conocimiento de la fisiopatología y de los medios de diagnóstico depende, en gran parte, la adecuada conducta terapéutica.

Muchas han sido las definiciones propuestas para este síndrome génito urinario; nosotros consideramos como más razonable la siguiente: La IUE es el escape involuntario de orina cuando la presión intravesical excede de la presión uretral máxima, pero sin que exista actividad del detrusor.

A las pacientes que consultaron por incontinencia urinaria se les hizo un test clínico con el cual se confirmó dicho diagnóstico.

Con el fin de descartar patología asociada a la IUE, se les practicó a todas las pacientes: Parcial de orina, Urocultivo, Calibración uretral, Cistoscopia y Prueba de Marshall. Descartada la patología asociada, quedaron para el estudio 31 pacientes, a las cuales se les solicitó uretrocistograma con cadena preoperatorio, con el fin de clasificar el tipo de IUE; y uretrocistograma postoperatorio para verificar la corrección de los ángulos uretrovesicales.

Este trabajo nos permitió concluir:

\section{PRIMERO:}

La necesidad del uretrocistograma con cadena preoperatorio, para la clasificación del tipo de incontinencia.

\section{SEGUNDO:}

La técnica quirúrgica a elegir, según la clasificación del tipo de incontinencia.

\section{TERCERO :}

La valoración del éxito o del fracaso de la intervención, de acuerdo con la corrección de los ángulos uretrovesicales anterior $\mathrm{y}$ posterior, demostrada por el uretrocistograma con cadena post-operatorio.

\section{SUMMARY}

The frecuency of feminine urinary stress in- continence is theme of constant actuality, since the knowledge of the pathophysiology and the proper diagnostic methods conduct, depend, in good part, to the adequate therapeutical management.

Many definitions have been propounded for the urinary stress incontinence; we consider the most exact definition as follows: URINARY STRESS INCONTINENCE is the involuntary escape of urine when intravesical pressure is greater then the highest uretral pressure, but without detrusor activity.

We effected a clinical test to our patients, which confirmed our dianostic. In order to laying away associated pathology, we effected them: Urine partial examination, Urography, Urethral size, Cistography Marshall proof.

Layed away associated pathology, we studied 31 patients, whom we search for one pre-surgical uretrocistography with chain, for the classification of the Urinary stress incontinence, and one post-surgical uretrocistography with chain for the verification of the uretrovesical angles correctness. This study gave us the following normas:

\section{FIRST:}

Necessity of the pre-surgical uretrocistography with chain for the classification of the Urinary stress incontinence.

\section{SECOND:}

Selection of the surgical technic according to the urinary stress incontinence type.

\section{THIRD:}

Its necessary to make the post-surgical uretrocistography with chain for the valuation of good accomplishment or failure of the surgery, by the verification of anterior and posterior urethrovesical angles correctness.

\section{BIBLIOGRAFIA}

1. BRADLEY, W.E. Control nervioso de la función uretrovesical. Clínicas ginecológicas y obstétricas. 3, 691, Septiembre 1978. 
2. GREEN, T. H. Jr. Urinary stress incontinence. Diferencial diagnosis, pathophysiology and management. Am. J. Obst. - Gynecol. 122, 368, 1975.

3. GREEN, T. H. Jr. Selección entre las vías de acceso vaginal o suprapúbica para el tratamiento quirúrgico de la incontinencia urinaria de alarma. Clínicas obstétricas y ginecológicas. 909, Diciembre 1977.

4. HARRISON, N.W. Incontinencia de esfuerzo, pasado y presente. Ginecología y obstetricia. Temas actuales. $1,65,1978$.

5. HODGKINSON, C. P. Uretrocistografía con cadenas de cuentas metálicas en la valoración pre y post operatoria de problemas urológicos ginecológicos. Clínicas Obstétricas y ginecológicas. 3, 763, Septiembre 1978.

6. HODGKINSON, C. P. Incontinencia de alarma recurrente. Clínicas Obstétricas y Ginecológicas. 3, 825, Septiembre 1978.

7. HURT, W. G. and J. A. FANTL. Uretrocistometría electrónica directa. Clínicas obstétricas y ginecológicas. 3, 733, Septiembre 1978.

8. KLUGO R. C. and J. C. CERNY. Cistometría standar electromiografía del esfiner uretral externo. Clínicas obstétricas y ginecológicas 3, 707, Septiembre 1978 .

9. LAZAREBKI M. Estudio radiológico de la incontinencia de esfuerzo en la mujer. Avances en obstetricia y ginecología $4,271,1978$.

10. KAZAREBKI M., A. LAZAROV, M. SAHPAZOV Y J. NAUMOV. Nuestras experiencias con el tratamiento quirúrgico de la incontinencia urinaria de esfuerzo en la mujer. Avances en obstetricia y ginecología. 4, 265. 1978.

11. LEES, D. H. Incontinencia urinaria de esfuerzo. Diagnóstico y selección de casos para tratamiento. Avances en obstetricia y ginecología. 4, 265. 1978.

12. LEE, R. A. Incontinencia recurrente de esfuerzo; valoración pre-operatoria $\mathrm{y}$ tratamiento quirúrgico. Clínicas obstétricas y ginecológicas. 19,661 , Septiembre 1978 .
13. MARSHALL, J. R. y G. E. JUDD. Guía para el tratamiento de mujeres con síntomas en vías urinarias bajas. Clínicas obstétricas y ginecológicas. 19, 247, Junio 1976.

14. MARSHALL, MARCHETTI, KRANTZ. The correction of stress incontinence by simple vesicourethral suspension. Surg. -Gynecol. -Obstet. $88 ; 509,1949$.

15. MEDINA, J, E. Incontinencia urinaria de esfuerzo en la mujer. Estudio radiológico. Revista Colombiana de Ginecologia y Obstetricia. Voi. XXI. No. 3 - 249. Mayo/Junio 1970.

16. MOLLER, C. F. Valoración: Parte I. Investigación de vías urinarias distales. Ginecología y obstetricia. Temas actuales. 1, 27-1978.

17. ROBERTSON, J. R. Uretroscopia con $\mathrm{C}^{02}$ en problemas urológicos ginecológicos. Clínicas obstétricas y ginecológicas. $3, \mathbf{7 7 5}$, Septiembre 1978 .

18. ROBERTSON, J. R. Valoración: Parte II. Uretroscopia con gas y estudios de la presión. Ginecología y obstetrica. Temas actuales 1, 39, 1978.

19. STANTON, S. L. Investigación pre-operatoria y diagnóstico. Clínicas obstétricas y ginecológicas. 3,743 , Septiembre 1978 .

20. STANTON S. L. Cirugía de la incontinencia u urinaria. Ginecología y obstetricia. Temas actuales. 1, 79. 1978.

21. SOLE, F.J.; J. CONEJERO; F. Jiménez; C. Rioja. Avances en Ginecología y Obstetricia. 4, 251,1978 .

22. SUNDBERG, A. I. Enfermedades de las vías urinarias. Avances en Ginecología y Obstetrica. 3, 755. 1975.

23. TANAGHO, E. A. Aspectos anatómicos y fisiológicos de la nicción. Ginecología y Obstetricia. Temas actuales. $1,3.1978$.

24. WILLIAMS, T. J. Incontinencia urinaria en la mujer. Clínicas Obstétricas y Ginecológicas. 729, Junio, 1974. 